\title{
Colonic Transit Study Technique and Interpretation: Can These Be Uniform Globally in Different Populations With Non-uniform Colon Transit Time?
}

TO THE EDITOR: We read with great interest the review article entitled "How to interpret a functional or motility test: colon transit study" by Kim et al. ${ }^{1}$ The authors reviewed colonic transit study using radio-opaque markers, scintigraphy and wireless motility capsule in evaluation of colonic motility. ${ }^{1}$ They described the method and criteria to differentiate between slow and normal transit constipation and fecal evacuation disorders. However, it is important to note that the method they described for assessment of colonic transit using radio-opaque markers, initially described by Hinton et al $^{2}$ as early as 1969 may not suit the population with rapid gut transit such as in India.

The authors referred to several studies in which either single or multiple capsule techniques were used. ${ }^{1}$ In single capsule technique, after ingestion of markers contained in capsules, several abdominal X-rays are performed until all markers are defecated or a single abdominal X-ray on day 5 (120 hours later). Similarly, in multiple capsule-technique, capsules are taken daily for 3 days followed by abdominal X-rays on day 4 and 7 or only on day 7 . In single capsule technique, when more than $20 \%$ of markers are retained, it is defined as delayed transit. ${ }^{3}$ Most of these series are from the West where mean colonic time was found to be 30-40 hours, with upper normal limit of 70 hours. ${ }^{4-8}$ However, this method was not found suitable in some Asian population especially in India where gut transit is fast.

Gut transit time is quite fast in Indian population. Though stool frequency of up to 3 per week is considered normal in Western population, ${ }^{9} 99 \%$ of Indians pass at least 1 stool per day. ${ }^{10}$ Average stool weight in 514 healthy Indians older than 15 years was $311 \mathrm{~g}$ per day. ${ }^{11}$ In contrast, stool weight greater than $200 \mathrm{~g}$ per day is diagnostic of diarrhea in Western population. ${ }^{12}$ Median mouth to cecum transit time was 65 minutes among 12 healthy Indians ${ }^{13}$ and total colonic transit time was 15.8 hours among 25 subjects. ${ }^{14}$ Considering fast gut transit among Indians, the protocol for assessment of colonic transit time using radio-opaque markers is likely to be different in this population.

When the Western protocol for assessment of transit time by administration of 20 radio-opaque markers each at 0,24 and 48 hours followed by an abdominal X-ray at 72 hours was used in Indian population, even at 72 hours, very few markers were found retained inside the abdomen due to rapid gut transit. ${ }^{10}$ Hence, attempts have been made to reduce the interval between ingestion of markers and abdominal X-ray. ${ }^{10}$ Nabar et $\mathrm{al}^{14}$ tried to administer markers initially according to the Western protocol; since most of the markers got expelled at 72 hours, they reduced the interval between administration of markers to 12 hours and then to 9 hours.

We performed colonic transit study ${ }^{15}$ using indigenously prepared radio-opaque markers (SGmark) in 9 patients with adult Hirschsprung's disease, 11 with chronic intestinal pseudo-obstruction and 11 healthy subjects. Twenty markers were administered each time at 0,12 and 24 hours followed by abdominal $\mathrm{X}$-rays at 36 and 60 hours. After 36 hours, total number of markers retained in the abdomen and those in the right segment in patients with Hirschsprung's disease and chronic intestinal pseudo-obstruction was higher than that in healthy subjects. In the abdominal X-ray at 60 hours, total number of markers and number in all segments were higher in patients with Hirschsprung's disease and chronic intestinal pseudo-obstruction than in healthy subjects. The best cut-off by receiver operating characteristic curves at 36 and 60 hours were 30 and 14 markers, respectively. The sensitivity, specificity, positive and negative predictive values, diagnostic accuracy and area under the receiver operating 
characteristic curve at 36 hours were $90 \%, 82 \%, 90 \%, 82 \%, 87 \%$ and 0.9 , respectively; the corresponding values at 60 hours were $95 \%, 100 \%, 100 \%, 92 \%, 97 \%$ and 0.99 , respectively. This study showed that an abdominal X-ray at 60 hours is better than that at 36 hours.

We conclude that colonic transit time varies markedly among different populations. It depends on race, ethnicity and dietary habits. The methods and normative data of one population may not be applicable to another population. Colon transit time should be standardized and validated for individual population. Our protocol of 12-hourly administration of markers should be followed for Indian population or other similar populations with rapid gut transit.

Uday C Ghoshal, Vikas Sengar and Deepakshi Srivastava Department of Gastroenterology, Sanjay Gandhi Postgraduate Institute of Medical Sciences, Lucknow, India

1. Kim ER, Rhee PL. How to interpret a functional or motility test - colon transit study. J Neurogastroenterol Motil 2012;18:94-99.

2. Hinton JM, Lennard-Jones JE, Young AC. A new method for studying gut transit times using radioopaque markers. Gut 1969;10: 842-847.

3. Evans RC, Kamm MA, Hinton JM, Lennard-Jones JE. The normal range and a simple diagram for recording whole gut transit time. Int J Colorectal Dis 1992;7:15-17.

4. Southwell BR, Clarke MC, Sutcliffe J, Hutson JM. Colonic transit studies: normal values for adults and children with comparison of radiological and scintigraphic methods. Pediatr Surg Int 2009;25:559572 .

5. Martelli H, Devroede G, Arhan P, Duguay C. Mechanisms of idiopathic constipation: outlet obstruction. Gastroenterology 1978;75: 623-631.
6. Meir R, Beglinger C, Dederding JP, et al. [Age- and sex-specific standard values of colonic transit time in healthy subjects.] Schweiz Med Wochenschr 1992;122:940-943. [German]

7. Metcalf AM, Phillips SF, Zinsmeister AR, MacCarty R, Beart RW, Wollff BG. Simplified assessment of segmental colonic transit. Gastroenterology 1987;92:40-47.

8. Sadik R, Abrahamsson H, Stotzer PO. Gender differences in gut transit shown with a newly developed radiological procedure. Scand J Gastroenterol 2003;38:36-42.

9. Adibi P, Behzad E, Pirzadeh S, Mohseni M. Bowel habit reference values and abnormalities in young Iranian healthy adults. Dig Dis Sci 2007;52:1810-1813.

10. Ghoshal UC, Abraham P, Bhatt C, et al. Epidemiological and clinical profile of irritable bowel syndrome in India: report of the Indian Society of Gastroenterology Task Force. Indian J Gastroenterol 2008;27:22-28.

11. Tandon RK, Prasad N, Gupta MC, Tandon BN. Stool weights and transit time in North Indians. J Assoc Physicians India 1976;24: 807-810.

12. Fine KD, Schiller LR. AGA technical review on the evaluation and management of chronic diarrhea. Gastroenterology 1999;116:14641486.

13. Ghoshal UC, Ghoshal U, Ayyagari A, et al. Tropical sprue?is associated with contamination of small bowel with aerobic bacteria and reversible prolongation of orocecal transit time. J Gastroenterol Hepatol 2003;18:540-547.

14. Nabar AA, Bhatia SJ, Abraham P, Ravi P, Mistry FP. Total and segmental colonic transit time in non-ulcer dyspepsia. Indian $\mathrm{J}$ Gastroenterol 1995;14:131-133.

15. Ghoshal UC, Gupta D, Kumar A, Misra A. Colonic transit study by radio-opaque markers to investigate constipation: validation of a new protocol for a population with rapid gut transit. Natl Med J India 2007;20:225-229.

\section{Conflicts of interest: None.}

